\title{
Lytic Efficiency of Mycobacteriophages
}

\author{
Sameer Hassan, Azger Dusthackeer, Balaji Subramanyam, C. Ponnuraja, Gomathi N. Sivaramakrishnan \\ and Vanaja Kumar
}

Tuberculosis Research Centre, Chetput, Chennai, India

\begin{abstract}
Mycobacteriophages D29 and TM4 are the two virulent phages widely used for the study of mycobacterial genetics. Both the phages are capable of killing Mycobacterium tuberculosis but the efficiency of these phages in killing has not been evaluated and compared. There are reports based on codon usage analysis where TM4 is predicted to be a better killing phage over D29 which corroborated with the whole genome in silico analysis. In addition a kill assay using 5 wild type virulent mycobacteriophages viz. D29, TM4, I3, Che7 and Che11 was performed to study the killing efficiency of these phages using LRP assay. Based on the results, D29 was found to infect all the 10 clinical strains of $M$. tuberculosis and significantly reduced RLU at 3 hours and this effect continued up to 24 hours. Thus, D29 is found to have better killing efficiency than TM4 contradicting the earlier predictions. In silico analysis of holin and lysin genes of TM4 and D29 substantiated our findings.
\end{abstract}

Keywords: Mycobacetriophages, codon usage, kill assay.

\section{INTRODUCTION}

The complex cell wall architecture of Mycobacterium tuberculosis defies the use of molecular tools in mycobacterial research. The discovery of mycobacteriophages, have facilitated the development of mycobacterial genetic systems and provided insights into viral diversity and the evolutionary mechanisms that generate them [1-5]. The resurgence of tuberculosis and emergence of drug resistance has stimulated fresh research on mycobacteriophages.

Genome sequences of 61 mycobacteriophages are available on online databases. The availability of these sequences has helped in understanding the evolutionary relationship and genetic diversity among these phages. The comparative genome analysis of 14 mycobacteriophages revealed that they are relatively large in genome size and contain large number of previously unidentified genes and are highly diverse at both the nucleotide and amino acid sequence levels [1]. The diversity at the nucleotide sequence level of mycobacteriophages appears to be greater than that of the diary phages [6] or staphylococcal phages [7]. Recently, Hatfull et al., has grouped 3357 proteins of 30 mycobacteriophage genomes into 1536 phamilies of related sequences that represent $50 \%$ of total number of phamilies in the mycobacteriophage population [8].

Mycobacteriophage TM4 is a virulent tailed-phage with double stranded DNA [9]. Its genome is approximately $50 \mathrm{~kb}$ in length and contains cohesive termini $[9,10]$. Mycobacteriophage D29 is a virulent phage, capable of infecting both fast and slow growing mycobacteria. It is a close relative of mycobacteriophage L5 with a $3.6 \mathrm{~kb}$

\footnotetext{
*Address correspondence to this author at the Department of Bacteriology, Tuberculosis Research Centre, Chetput, Chennai-600 031, India; Tel: +9144-28369659; Fax: +91-44-28362528;

E-mail: vanajakumar@trcchennai.in
}

deletion removing the repressor gene that accounts for the inability of D29 to form lysogens [11]. TM4, D29 along with L5 and BxZ2 are identified to have the broadest host range capable of forming plaques on all of the slow growing species [12]. The ability of D29 to enter and kill macrophages infected with $M$. tuberculosis was reported earlier whereas TM4 cannot enter macrophage on its own due to lack of specific receptors. D29 genome might encode specific protein receptors which are recognized by the macrophage facilitating the phagocytosis of D29 [13].

Study of codon usage in fourteen mycobacteriophage genomes revealed the role of mutational pressure at the third synonymous codon position and translation selection acting in highly expressed genes [14]. Based on another study on codon usage of six lytic mycobacteriophage genomes, the role of mutational and translational forces acting on these genomes were determined [15]. The authors further identified that Che9c, BxZ1 and TM4 may be extremely virulent phages as majority of their genes have high translation efficiency.

In the current study, we have analyzed the killing efficiency of five lytic mycobacteriophages using in vitro methodology and further corroborated with bioinformatics analysis.

\section{MATERIAL AND METHODS}

\section{Sequence}

The protein coding sequences of D29 and TM4 were downloaded from GenBank. Genes were selected based on the following criteria: (a) genes having $\geq 300$ bases (b) genes having proper start and stop codons (c) genes with no stop codons within.

\section{Bioinformatics}

Sequence analysis: PHI- Blast (http://blast.ncbi.nlm. nih.gov/Blast.cgi) was used for database search. 
TMHMM and SignalP were used for the prediction of transmembrane helices and signal peptide respectively. Plotting of hydrophobicity profile was done with Bioedit software. ClustalW was used for multiple sequence alignment while PFAM and InterPro were made use of for domain analysis.

(b) Codon usage analysis was performed for the protein coding sequences of TM4 and D29. The relative synonymous codon usage (RSCU) in both phages was determined to study the overall codon usage variation among the genes and genomes. RSCU is defined as the ratio of the observed frequency of codons to the expected frequency if all the synonymous codons for those amino acids are used equally [16]. RSCU values greater than 1.0 indicates that the corresponding codons are more frequently used than expected and vice versa. GC3S is the frequency of $(\mathrm{G}+\mathrm{C})$ and $\mathrm{A} 3 \mathrm{~S}$, $\mathrm{T} 3 \mathrm{~S}, \mathrm{G} 3 \mathrm{~S}$, and $\mathrm{C} 3 \mathrm{~S}$ are the frequencies of $\mathrm{A}, \mathrm{T}, \mathrm{G}$, and $\mathrm{C}$ at the synonymous third positions of codon. These frequencies were calculated for the genes of both the genomes. $\mathrm{Nc}$, the effective number of codons used by a gene is generally used to measure the bias of synonymous codons and it is independent of amino acid compositions and codon number [17]. The values of Nc range from 20 (when one codon is used per amino acid) to 61 (when all the codons are used with equal probability). Highly biased genes are generally highly expressed [18]. As there is no information of gene expression level of bacteriophages, we have considered the highly biased genes as highly expressed in the case of mycobacteriophages under study. Hence the sequences in which the Nc values were less than 35 and those having $\mathrm{Nc}$ values greater than 55 were considered to be highly and lowly expressed respectively. Correspondence analysis using RSCU values were performed. CodonW 1.3 (http://www.molbiol.ox.ac.uk/cu) was used for correspondence analysis [19] and for analyzing the above mentioned parameters.

\section{Phages and Bacteria}

A total of ten clinical strains of Mycobacterium tuberculosis isolated at Tuberculosis Research Centre, Chennai were grown at $37^{\circ} \mathrm{C}$ on Lowenstein-Jensen (LJ) slopes. Suspensions of these strains equivalent to McFarland's standard \# 2 were prepared in Middlebrook 7H9 (Difco) supplemented with Bovine Albumin-Dextrose complex and $0.5 \%$ glycerol. Each suspension was divided into 6 aliquots of $1.5 \mathrm{ml}$ each to which $300 \mu 1$ of phages namely D29, TM4, I3, Che7, Che11 at a titer of $1 \times 10^{10} \mathrm{pfu} / \mathrm{ml}$ was added. Mycobacteriophage buffer was used as a negative control.
After 3 and 24 hours of incubation, the number of tubercle bacilli remaining alive was determined by performing a luciferase reporter phage assay. This was done by adding 50 $\mu \mathrm{l}$ of luciferase reporter phage phAE129 $\left(1 \times 10^{10} \mathrm{pfu} / \mathrm{ml}\right)$ to $250 \mu \mathrm{l}$ of initial wild type phage-cell mixture and incubating for 4 hours. Light output was measured as relative light units (RLU) using the luminometer (Monolight 2010) with 10 seconds integration time after adding $0.33 \mathrm{mM}$ D-luciferin (R\&D Systems, Minneapolis) in $0.05 \mathrm{M}$ sodium citrate buffer (pH 4.5) to phage-cell mixture. Reduction in RLU by $50 \%$ compared to the buffer control was considered to reflect significant reduction in viability.

\section{RESULTS}

\section{Gene Organization}

The genome of D29 and TM4 is $49 \mathrm{~kb}$ and $52 \mathrm{~kb}$ long respectively. Both the genomes have two lysin and one holin coding genes in their genomes. Double stranded DNA bacteriophages use the dual system i.e endolysin-holin to achieve lysis of their bacterial host. TM4 and D29 being lytic mycobacteriophages possess the dual lytic system to lyse the host cell. Comparison of the organization of endolysin-holin genes in TM4 and D29 genomes (Fig. 1) revealed holin gene being sandwiched between two lysin genes in D29 whereas in TM4, holin was found to be present at the end of the two lysin genes.

\section{Codon Usage Analysis}

The overall GC content of TM4 and D29 was found to be 68.7 and 63.78 respectively. As both the phages carry GCrich genomes, codons ending in $\mathrm{G}$ and $\mathrm{C}$ should be predominant in the coding sequences. It was observed that the Nc values for TM4 and D29 ranged from 27.79 to 44.42 and 29.38 to 59.2 respectively, whereas the GC3s values ranged from 71.2 to 95.3 and 63.5 to 93.3 respectively. This showed high variation in the $\mathrm{Nc}$ and $\mathrm{GC} 3 \mathrm{~s}$ values among the genes of both the genomes.

Nc plots (a plot of Nc versus GC3s) and correspondence analysis (CA) determine the determinants of the codon usage variations in different organisms. The Nc plot for the genes of both TM4 and D29 phages showed that majority of the genes lay below the expected curve towards GC rich regions suggesting that these phages displayed an additional codon usage bias which was independent of GC3s (Fig. 2).

Apart from mutational bias, translational bias also plays a major role in shaping the codon usage of these phages. A scatter plot was drawn between the position of genes along the first major axis and $\mathrm{Nc}$ values (Fig. 3). Majority of the TM4 genes were found to have lower Nc values than D29.
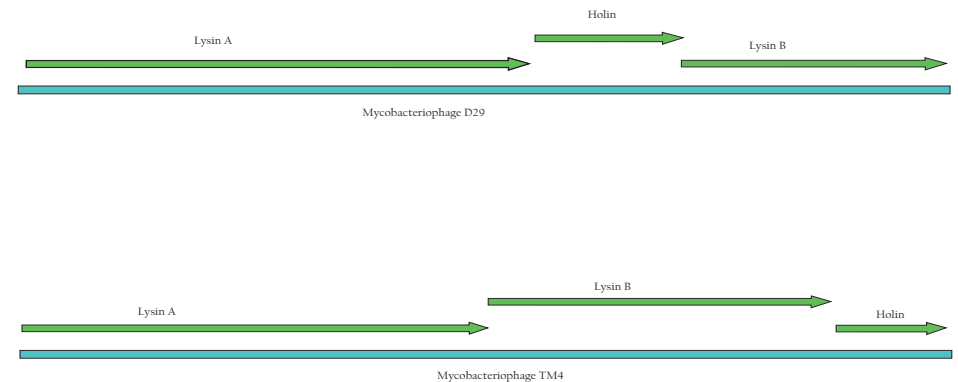

Fig. (1). Organization of lysin and holin genes in the genome of D29 and TM4. 


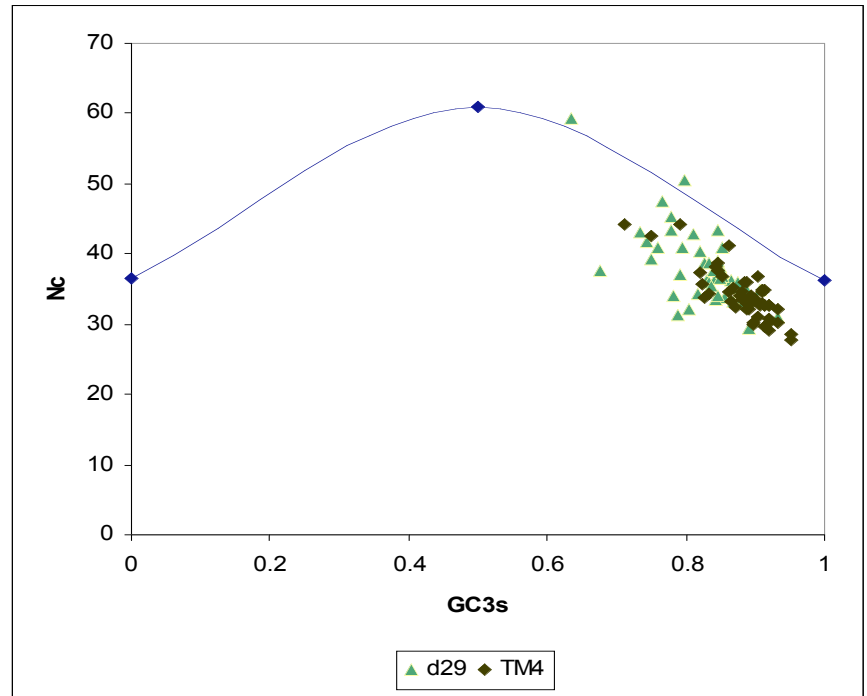

Fig. (2). Nc plot of genes from D29 (green) and TM4 (black).

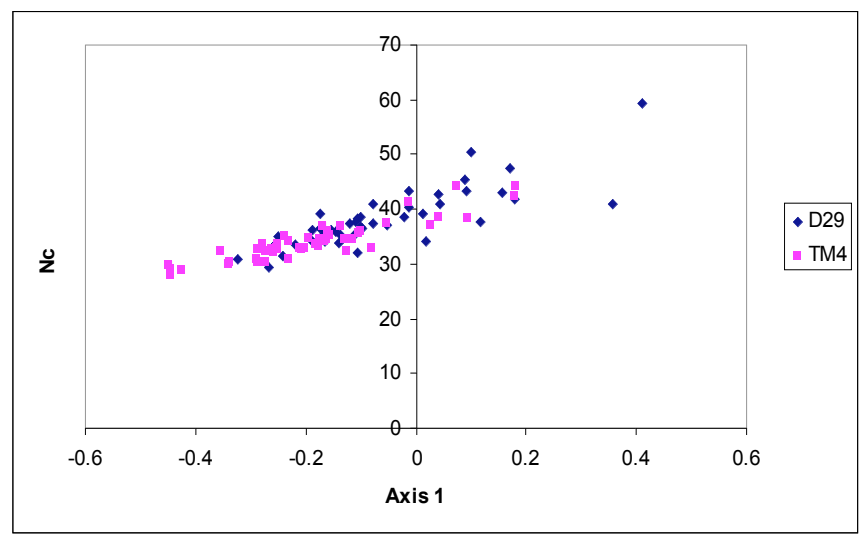

Fig. (3). Scatter plot of the genes of D29 (blue) and TM4 (pink) against Nc values.

Correspondence analysis showed the distribution of the genes of the two genomes along the first two major axes, accounting for $12.1 \%$ and $8.3 \%$ respectively of the total variation. Based on the results obtained (data not shown) there were 5 over-represented codons in the genes located at the negative side of the first major axis. Out of 5 predominant codons, 4 ended in $\mathrm{C}$ and the other ended in $\mathrm{G}$ at the third position.

A comparative analysis of the positions of genes along first major axis and the nucleotide composition at the third codon position revealed that the first axis was positively correlated with A3 and T3 but negatively correlated with G3 and $\mathrm{C} 3$ (Table 1). The results suggested that $\mathrm{A}$ and $\mathrm{T}$ ending codons were clustered on the positive side whereas $\mathrm{G}$ and $\mathrm{C}$ ending codons were located on the negative side of first major axis.

Table 1. Correlations Coefficient Between the Positions of Genes Along the First Major Axes with Different Parameters

\begin{tabular}{|c|c|c|c|c|c|c|c|}
\hline Axis & A3s & T3s & G3s & C3s & GC & GC3s & Nc \\
\hline First & 0.667 & 0.582 & -0.292 & -0.429 & -0.693 & -0.773 & 0.728 \\
\hline
\end{tabular}

\section{Kill Assay}

Among the five phages tested D29 was found to be most effective in producing more than $50 \%$ killing in all the ten strains at 3 and 24 hours (Fig. 4). Che 7 exhibited similar efficiency in 8 strains at 24 hours, whereas TM4 was the least effective among the phages tested. It was able to produce more than $50 \%$ killing in only 3 of the clinical isolates.

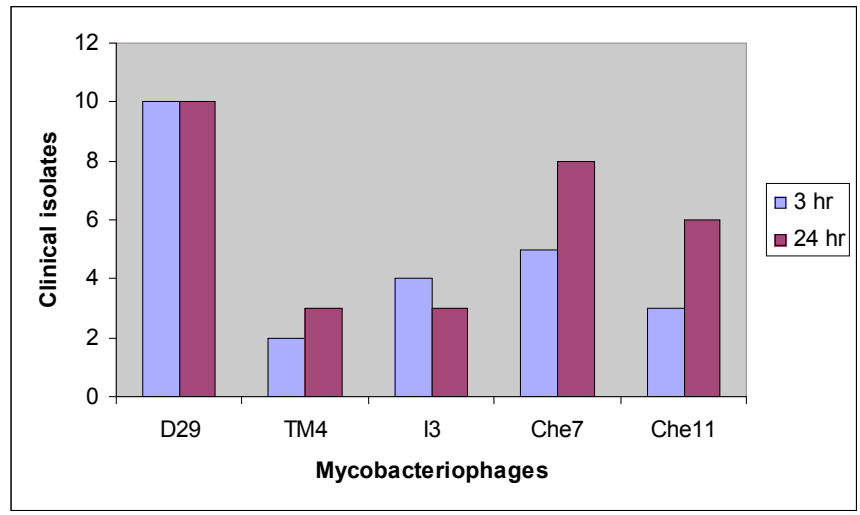

Fig. (4). Lytic activity of phages in clinical strains of Mycobacterium tuberculosis.

\section{Sequence Analysis of Holin and Lysin}

\section{Nc Values for Lysin and Holin}

Apart from overall $\mathrm{Nc}$ values for both the genomes, $\mathrm{Nc}$ values for lysin and holin genes were compared separately. Based on the Nc values (Table 2) of lysin and holin of both genomes, the lysin gene of TM4 showed lower Nc value than that of D29, whereas holin gene of D29 showed lower Nc value than of TM4.

Table 2. Nc Values for Lysin A, Lysin B and Holin Genes of D29 and TM4

\begin{tabular}{|c|c|c|c|}
\hline Phage & Lysin A & Lysin B & Holin \\
\hline \hline TM4 & 35.27 & 34.02 & 34.3 \\
\hline D29 & 38.66 & 40.93 & 33 \\
\hline
\end{tabular}

\section{Sequence Analysis of Lysin and Holin of TM4}

Gp29 and Gp30 of TM4 are identified to code for lysin protein and are 547 and 400 amino acid long respectively. Based on the prediction of phage genes into gene families by Hatfull et al. for 30 mycobacteriophage genome, Pham7 contains Lysin A genes and Pham9 contains Lysin B genes [6]. In the current study GP29 of TM4 was identified as Lysin A and GP 30 as Lysin B as predicted earlier.

Lysin A protein of TM4 was recognized to have Nacetylmuramoyl-L-alanine amidase domain (209-356) based on PFAM analysis. Blast search using Lysin A protein of TM4 identified significant similarity to N-acetylmuramoylL-alanine amidase protein encoded by Mycobacterium sp. MCS (58\% with aa). GP29 of TM4 was therefore designated as N-acetylmuramoyl-L-alanine amidase. Multiple sequence alignment of GP29 with other known 


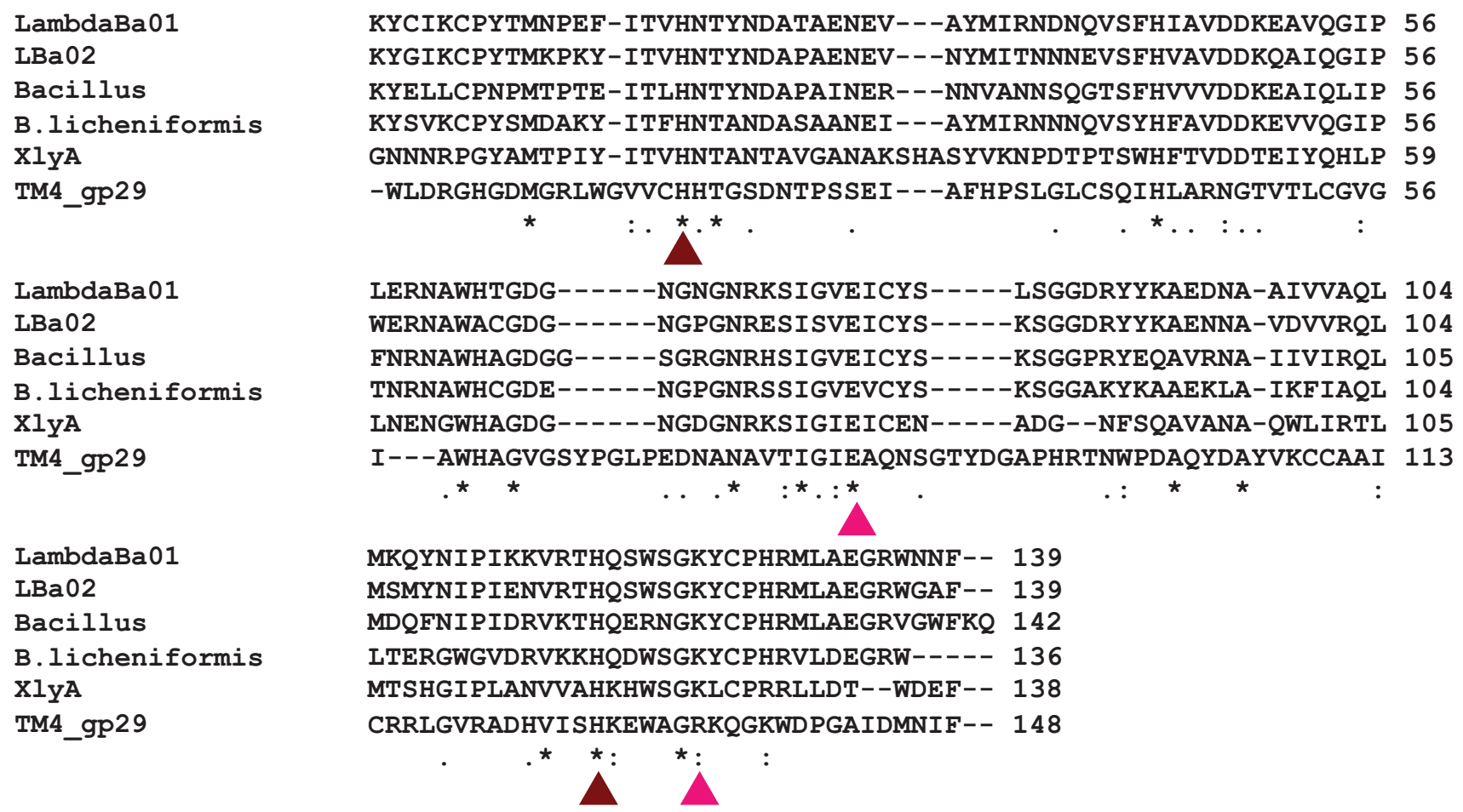

Fig. (5). The residues involved in zinc binding and catalytic triad are conserved among the Bacillus endolysins and GP29 of TM4. These are indicated by brown and pink triangles, respectively. Bacillus and XlyA are endolysins from Bacillus phage TP21-L and B. subtilis respectively.

amidase proteins revealed the conservity of zinc binding and catalytic triad residues (Fig. 5).

Lysin B protein encoded by GP30 was identified to have a peptidoglycan binding domain at the $\mathrm{N}$-terminal region of GP30. Blast search and PHYRE using Lysin B of TM4 as query sequence identified similarity to $\alpha / \beta$ hydrolases. Multiple alignment of Lysin B sequences of mycobacteriophages identified the presence of a conserved pentapeptide Gly-Tyr-Ser-Gln-Gly motif at positions 162166 of the Lysin B amino acid sequence matching with the characteristic Gly-X-Ser-X-Gly motif found in lipolytic enzymes (Fig. 6).

GP31 of TM4 codes for holin protein of 128 amino acids consisting of two transmembrane helices with highly charged $\mathrm{N}$ terminal and $\mathrm{C}$ terminal (Fig. 7).

\section{Sequence Analysis of Lysin and Holin of D29}

GP 10 and GP 12 of D29 codes for Lysin A (493 aa) and Lysin B (254 aa) respectively. Lysin A was identified to have Lysozyme like domain (198-362). The Gly-X-Ser-XGly motif found in lipolytic enzymes along with the residues involved in the catalytic triad was identified to be well conserved in GP 12 (lysin B) protein sequence of D29 (Fig. 6).

GP11 of D29 codes for holin protein belonging to Class II holin. Two transmembrane helices were predicted using TMHMM. GP11 was found to have a short charged $\mathrm{N}$ terminal region and a highly charged $\mathrm{C}$ terminal region (Fig. 8).

\section{DISCUSSION}

In the current study, the codon usage of D29 and TM4 genomes and $\mathrm{Nc}$ values for holin and lysin genes coded by both genomes were analysed and compared. Based on the $\mathrm{Nc}$ plot, apart from the mutational force, some other factors are also understood to play an important role in shaping the codon usage in both the genomes. Identification of significant positive correlation between Nc values and Axis 1 revealed that substantial number of TM4 genes had lower $\mathrm{Nc}$ values as compared to D29 genes. On the basis of this result it is predicted that a balance between mutational force and selection due to translational efficiency is strongly operating in selecting the codon usage variation among the genes of these phages. The number of highly biased genes were more in TM4 (72\%) than in D29 (30\%) genome. Several reports have shown earlier that synonymous codon usage in the highly expressed genes of diverse array of organisms is influenced by cellular tRNA abundance [2025]. Based on the comparative analysis between $M$. tuberculosis $\mathrm{H}_{37} \mathrm{Rv}$ tRNA and synonymous codon usage, it is identified that all the over represented codons of D29 and TM4 are recognized by host tRNA. On the basis of our analysis it is expected that more number of TM4 genes should be efficiently translated leading to better lytic activity and faster killing. Earlier report by Ranjan et al. [15] predicting the genes of TM4 to be expressed rapidly by the host's translation machinery corroborated with our finding.

The better translational efficiency of TM4 was expected to quicken the lysis process qualifying it to be a better lytic 
gp12_D29 gp52 Wildcat gp10 Lockley gp11_KBG gp 8 Ū2 gp11 DD5 gp11_Jasper gp9_Bxb1 gp $1 \overline{0}$ Solon gp9_Bethlehem gp241_Catera gp238 Bxz1 gp242 Cali gp31_Llij gp31-Pacc40 gp33 Boomer gp34_Ramsey gp3_Ms 6 TM4_gp 30

gp12_D29 gp52_Wildcat gp10_Lockley gp11_KBG gp 8 U 2 gp1 1 DD5 gp11_Jasper gp 9 Bxb1 gp10 Solon gp9_Bethlehem gp $2 \overline{4} 1$ Catera gp238 Bxz1 gp242_Cali gp31_Llij gp31_Pacc40 gp33 Boomer gp34_Ramsey gp3_Ms 6 TM4_gp 30

gp12_D29 gp52_Wildcat gp10_Lockley gp11_KBG gp 8 Uे2 gp11_DD5 gp11_Jasper gp9_Bxb1 gp10_Solon gp9_Bethlehem gp $2 \overline{4} 1$ Catera gp238 Bxz1 gp242_Cali gp31_Llij gp31_Pacc40 gp33_Boomer gp34_Ramsey gp3_M̄s 6 TM4_gp30
-ADPYADFAMA GYSQGA IVVGQVLKHHILPPTGRLHRFLHRLKKVIFWGNPMRQKGFAHS 128 -----GKFTLA GYSQGAMVTS IVYKYDLLDPMGRLHHRLPDFMGGVTWGNPMREMGKAWT 203 RRIERYGLSMIGYSQGAIVTSELWEYHIKPVTGRLHWVKDHVRGAVTFGNPMRETGKVWP 204 RRIERYGLSMIGYSQGAIVTSELWEYHIKPVTGRLHWAKDHVRGAVTFGNPMRETGKVWP 204 QRIERYGLSMIGYSQGAIVTSELWEYHIKPVTGRLHWVKDHVRGAVTFGNPMRETGKVWP 204 QRIERYGLSMIGYSQGAIVTSELWEYHIKPVTGRLHWVKDHVRGAVTFGNPMRETGKVWP 204 QRIERYGLSMI GYSQGAIVTSELWEYHIKPVTGRLHWVKDHVHGAVTFGNPMRETGKVWP 204 RRIERYGLSMI GYSQGAIVTSELWEYHIKPVTGRLHWVKDHVRGAATFGNPMRETGKVWP 204 QRVERYGLSMIGYSQGAIVTSELWEYHIKPVTGRLHWVKDHVRGAVTFGNPMRETGKVWP 204 QRIERYGLSMIGYSQGAIVTSELWEYHIKPENGRLHWVKDHVRGAATFGNPMREIGKVWP 204 KQVERNGMVLA GYSQGAIVTSEVWEDDIRTSGPIVGWAKDHVLKAVAWGNPNREQGKAYP 225 KQVERNGMVLA GYSQGAIVTSEVWEDDIRTSGPIVGWAKDHVLKAVAWGNPNREQGKAYP 225 KQVERNGMVLAGYSQGAIVTSEVWEDDIRTSGPIVGWAKDHVLKAVAWGNPNREQGKAYP 225 QRIETHGAALA GYSQGAVVVSELWMNHIAPETGSLHWMKPHIEKAVTWGNPNRELGHVWA 215 QRIETHGAALA GYSQGAVVVSELWMNHIAPETGSLHWMKPHIEKAVTWGNPNRELGHVWA 215 ERIETHGAALA GYSQGAVVVSELWMNHIAPETGSLHWMKPHIEKAVTWGNPNRELGHVWA 215 ERIETHGAALA GYSQGAVVVSELWMNHIAPEDGSLHWMKPHIEKAVTWGNPNRELGHVWA 220 ERIETHGTALA GYSQGAVVLSELWMNHIAPEDGSLRWMKPHVRKAVTWGNPNRELGHVWA 214 -ARDFDYIVLIGYSQGALPASRLMRRILS---GDLQRFKSKLIAGVTFGNPMREKGHTFP 207

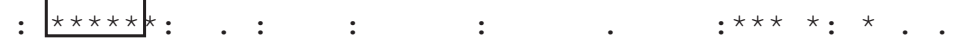

DEWIHPVAAPDTLGILEDRLENLEQYGFEVRDYAHDGDMYASIKEDDLHEY---EVAIGR 185 DG-VGAVAGQNNGGIAEDRLVGTP---WNWRDYAHKGDLYTDCEFDDEGEY---KRSVCK 256 DPG-GQMPSAKSHGIADQLMVDTPD---WWRNYAHKGDLYTDCE-GDSGEN---KTAIYK 256 DPG-GQMPSAKSHGIADQLMVDTPD---WWRNYAHKGDLYTDCE-GDSGEM---KTAIYK 256 DPG-GQMPSAKSHGIADQLMVDTPD---WWRNYAHKGDLYTDCE-GDSGEM---KTAIYK 256 DPG-GQMPSAKSHGIADQLMVDTPD---WWRNYAHKGDLYTDCE-GDSGEM---KTAIYK 256 DPG-GQMPSAKSHGIADQLMVDTPD---WWRNYAHKGDLYTDCE-GDSGEM---KTAIYK 256 DPG-GQMPSAKSHGIADQLMVDTPD---WWRNYAHKGDLYTDCE-GDSGEM---KTAIYK 256 DPG-GQMPSAKSHGIADQLMVDTPD---WWRNYAHKGDLYTDCE-GDSGEM---KTAIYK 256 DPG-GQLPSAKSQGIADQLMGDTPD---WWRNYAHKGDLYTDCE-GDSGEM---KTAIYK 256 DAG-APLAAADSAGITGRLMTDTPD---WWRNYAHQGDLYTATRPGESRED---KVAIWQ 278 DAG-APLAAADSAGITGRLMTDTPD---WWRNYAHQGDLYTATRPGESRED---KVAIWQ 278 DAG-APLAAADSAGITGRLMTDTPD---WWRNYAHQGDLYTATRPGESRED---KVAIWQ 278 DHGGSPMAPSNTQGVSSNGMRDTPP---WWRDYAHQGDLYACTEPGDTQEV---RNAIWQ 269 DHGGSPMAPSNTQGVSSNGMRDTPP---WWRDYAHQGDLYACTEPGDTQEV---RNAIWQ 269 DHGGSPMAPSNTQGVSSNGMRDTPP---WWRDYAHQGDLYACTEPGDTQEV---RNAIWQ 269 DHGGSPMAPSNTQGVSSNGMRDTPG---WWRDYAHQGDLYACTEPGDTQEV---RNAIWQ 274 DHGGSPMAPSNTQGVSSNGMRNTPD---WWRDYAHQGDLYACTEPGDTQEV---RNAIWQ 268 GG-----ADPGGHGLDPQCLVNTPD---WWHDYAAKGDIYTVGSGSNDEKANADMTFIYQ 259 . $\quad{ }^{\star}: \quad:$. $\quad::^{\star \star} .{ }^{\star \star}:{ }^{\star}: \quad .: \quad: \quad$ :

-FIGGRDSVVAQLIELGQRPITEGIALAGAIIDALTFFARSRMGDKWPHL-YNRYP---- 246 -VFGGPDSILRQVIELGLDPFGEAIPMIKAISDAGMFFINRTT----PHINYNVGP---- 313 -VFSGPDSILRQLLEIGVNPTFELIALIRAVLDAGLFFIRGTT----PHTNYNIDP---- 313 -VFSGPDSILRQLLEIGVNPTFELIALIRAVLDAGLFFIRGTT----PHTNYNIDP---- 313 -VFSGPDSILRQLLEIGVNPTFELIALIRAVLDAGLFFIRGTT----PHTNYNIDP---- 313 -VFSGPDSILRQLLEIGVNPTFELIALIRAVLDAGLFFIRGTT----PHTNYNIDP---- 313 -VFSGPDSILRQLLEIGVNPTFELIALIRAVLDAGLFFIRGTG----PHVNYNIDP---- 313 -VFSGPDSILRQLLEIGVNPTFELIALIRAVLDAGLFFIRGTG----PHVNYNIDP---- 313 -VFSGPDSILRQLLEIGVNPTFELIALIRAVLDAGLFFIRGTG----PHVNYNIDP---- 313 -VFSGPDSILRQLLEIGVNPTFELIALIRAVLDAGLFFIRGTT----PHTNYNIDP---- 313 -ILSGPDSLLRQFLEIAEAPVPNAIAAFQAFMDAGLFFVKGTR----PHTNYHIGA---- 335 -ILSGPDSLLRQFLEIAEAPVPNAIAAFQAFMDAGLFFVKGTR----PHTNYHIGA---- 335 -ILSGPDSLLRQFLEIAEAPVPNAIAAFQAFMDAGLFFVKGTL----PHTNYHIGA---- 335 -LFTGPDSLLAQVIELVQAPLPETIAITKAILDAGMFFAKRTG----PHVDYNVQP---- 326 -LFTGPDSLLAQVVELVQAPLPEIIAITKALLDAGMFFAKRTG----PHVDYNVQP---- 326 -LFTGPDSLLAQVVELVQAPLPEAIAITKALLDAGMFFAKRTG----PHVDYNVQP---- 326 -LFTGPDSLLAQVIELVQAPLPETIAITRAILDAGMFFAKRTG----PHVDYNPQP---- 331 -LFTGPDSLLAQVIELAQAPLPETIAITRAILDAGMFEAKRTG----PHVDYNPQP---- 325 ALTDHQRGLVEAVLALLANPFAEVPAAVKAIVSGVGFIATNPP--TAPHIEYHIREAAPG 377

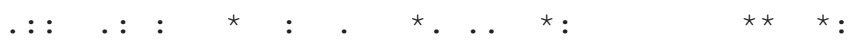

Fig. (6). Multiple sequences alignment of Lysin B protein sequence of mycobacteriophages using ClustalW. Identical (*), highly similar (:) and similar (.) amino acids are indicated. The conserved pentapeptide is boxed. The conserved Asp and His residues are indicated in bold. 


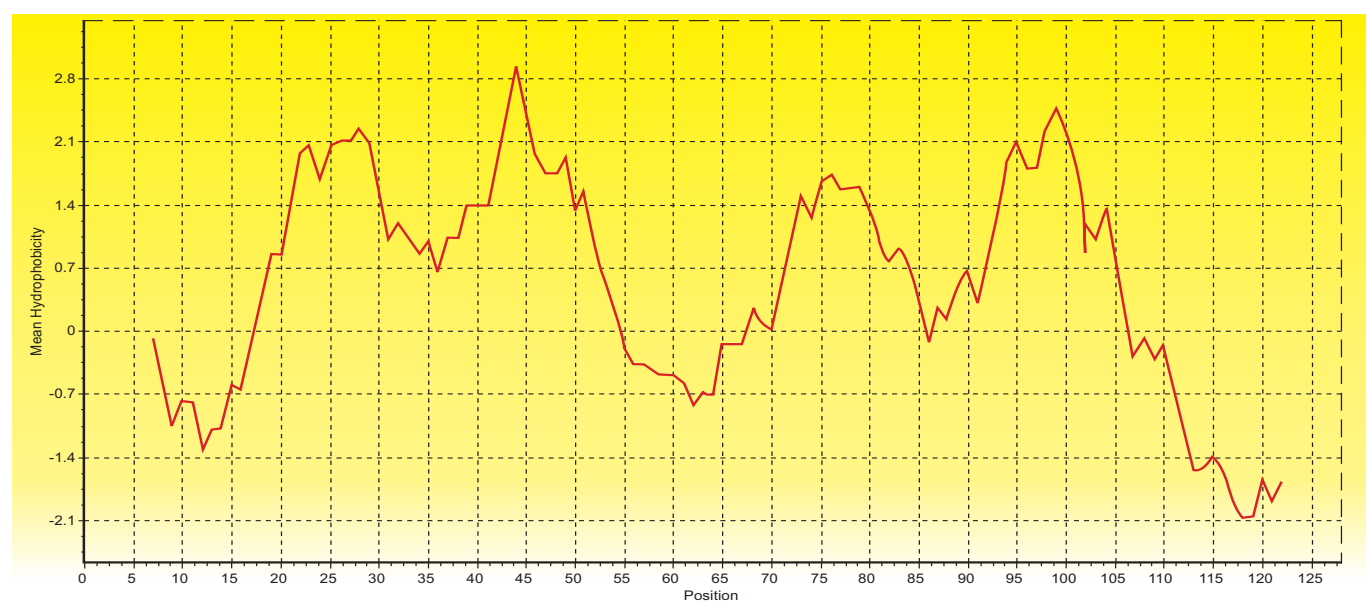

Fig. (7). Hydropathy plot of Holin protein of TM4.

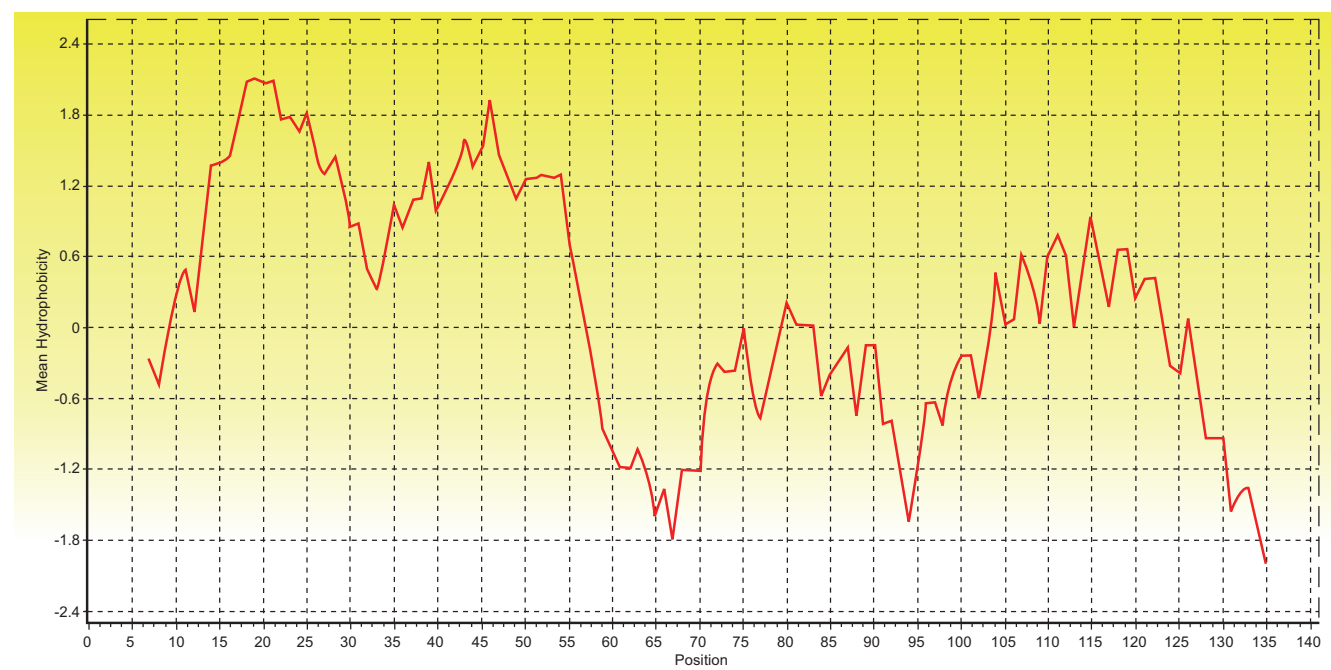

Fig. (8). Hydropathy plot of Holin protein of D29.

phage. However, the lytic performance of TM4 was poor in all the ten strains tested in vitro whereas D29 was efficient in effectively killing all of them at 3 hours and that effect was observed to continue up to 24 hours (Fig. 4).

Subsequently, holin and lysin genes of both the phages were analysed in detail to understand the performance of D29 over TM4 on the clinical strains of M. tuberculosis. Holin sequences of TM4 (Class I holin) and D29 (Class II holin) were found to be highly divergent sharing no amino acid similarity. Holin of TM4 was identified to be longer possessing a highly charged $\mathrm{N}$ terminal region whereas holin of D29 was shorter possessing a lowly charged $\mathrm{N}$ terminal region. Presence of highly charged N-terminal regions in holins has been reported to be a typical feature of holininhibitors [26]. Thus, it is likely that the presence of the highly charged $\mathrm{N}$ terminal region of holin in TM4 acted as antiholin thereby delaying the lysis process of TM4.

Virulent phages contain more tRNAs than temperate ones. They have higher codon usage biases and compositional differences with respect to the host genome. Even though phages use most of the cell's translation machinery, they can complement it with their own genetic information to attain higher fitness [27]. The presence and expression of phage tRNA genes that are already present in the host genome will have additional benefit on the phage genome [27]. So the presence of 5 tRNA genes in D29 genome compared to none in TM4 should further increase the fitness of translational efficiency of the genes of D29, allowing the expression of low biased genes in the genome. Moreover among the over-represented codons of TM4 phage few were recognized by the abundant host tRNAs compared to D29.

The cell wall of mycobacteria has a high lipid content which is absent in other gram positive bacteria. Mycobacterial cell envelop consists of long-chain mycolic acids esterified to an arabinogalactan polysaccharide, which is attached to the peptidoglycan backbone. This mycolylarabinogalactan-peptidoglycan complex (core) intercalates with an array of unusual free lipids, resulting in an effective external permeability barrier [28, 29]. The presence of additional lysis genes such as $\mathrm{Rz}$ and Rzl was reported earlier in bacteriophages enabling the efficient infection of Gram negative bacteria $[30,31]$. Similarly, the presence of mycolic acid in mycobacterial cell wall may require additional lysis genes in order to dislocate the complex mycolic acid. The lysin coding genes of mycobacteriophages had been organized into phamilies such as Pham 7 and Pham 
9 of related sequences [8]. Pham 7 contains lysin A genes, one of the two lysins of mycobacteriophages and Pham 9 contains lysin B genes. Lysin B in the genomes of D29 and TM4 were identified to have the conserved Gly-X-Ser-XGly motif of lipolytic enzyme. Based on Blast analysis, it was found that Lysin B gene is present exclusively in mycobacteriophage genomes probably enabling the infection of M. tuberculosis effectively.

Multiple sequence alignment of Lysin B of mycobacteriophages revealed the conservity of Gly-X-SerX-Gly motif characteristic of lipolytic enzymes in 19 of them (Fig. 6). However the amino acid sequence of Lysin B did not show characteristic features of any of the lipase families identified by Arpigny \& Jaeger [32-33]. Based on profile-profile matching algorithm of PHYRE, lysin B was identified to show similarity to cutinase like family of proteins. An important feature of the $\alpha / \beta$ hydrolase superfamily is the presence of Asp and His residues which form the catalytic triad together with the Ser residue [32, 3435]. LysB protein of Ms6 mycobacteriophage was reported to belong to the family of serine hydrolases [31] as is the case for lipid hydrolases [34]. The alignment (Fig. 6) showed that Asp and His residues were conserved among the homologous proteins of the mycobacteriophages. Although three Asp residues (Asp129, Asp166, Asp224) of D29 lysin B were totally conserved, Asp129 together with His163 might be good candidates for the catalytic triad, obeying the order Ser- Asp-His. However, in GP30 of TM4, Asp237 (Asp166 in D29) was conserved but Asp129 and His 163 in D29 are replaced by Gly and Ala in TM4 respectively. These amino acid substitutions may probably influence the activity of Lysin B in TM4 which is not yet experimentally proved.

Though over all codon bias of TM4 genome was stronger than that of D29, the details of lysis cassettes and presence of tRNAs of both the phages revealed that D29 was well equipped to efficiently lyse the host cells faster. The killing efficiency of D29 compared to four other lytic phages including TM4, as observed in the kill assay resulted in loss of viability of $M$. tuberculosis clinical strains as early as 3 hours. Thus, predictions by bioinformatics approach and laboratory experiments mutually contributed to each other quickening the understanding of the biological processes.

\section{ACKNOWLEDGEMENTS}

This work was financially supported by ICMRBiomedical Informatics Centre. We also acknowledge K. Ramakrishnan and G. Vadivu for their technical assistance.

\section{REFERENCES}

[1] Pedulla ML, Ford ME, Houtz JM, et al. Origins of highly mosaic mycobacteriophage genomes. Cell 2003; 113(2): 171-82.

[2] Hatfull GF, Sarkis GJ. DNA sequence, structure and gene expression of mycobacteriophage L5: a phage system for mycobacterial genetics. Mol Microbiol 1993; 7(3): 395-405.

[3] Mediavilla J, Jain S, Kriakov J, et al. Genome organization and characterization of mycobacteriophage Bxb1. Mol Microbiol 2000; 38(5): 955-70

[4] Ford ME, Sarkis GJ, Belanger AE, Hendrix RW, Hatfull GF. Genome structure of mycobacteriophage D29: implications for phage evolution. J Mol Biol 1998; 279(1): 143-64.

[5] Ford ME, Stenstrom C, Hendrix RW, Hatfull GF. Mycobacteriophage TM4: genome structure and gene expression. Tuber Lung Dis 1998; 79(2): 63-73.
[6] Brussow H. Phages of dairy bacteria. Annu Rev Microbiol 2001; 55: 283-303.

[7] Kwan T, Liu J, DuBow M, Gros P, Pelletier J. The complete genomes and proteomes of 27 Staphylococcus aureus bacteriophages. Proc Natl Acad Sci USA 2005; 102(14): 5174-9.

[8] Hatfull GF, Pedulla ML, Jacobs-Sera D, et al. Exploring the mycobacteriophage metaproteome: phage genomics as an educational platform. PLoS Genet 2006; 2(6): e92.

[9] Timme TL, Brennan PJ. Induction of bacteriophage from members of the Mycobacterium avium, Mycobacterium intracellulare, Mycobacterium scrofulaceum serocomplex. J Gen Microbiol 1984; 130(8): 2059-66.

[10] Jacobs WR, Jr., Tuckman M, Bloom BR. Introduction of foreign DNA into mycobacteria using a shuttle phasmid. Nature 1987; 327(6122): 532-5.

[11] Gomathi NS, Sameer H, Kumar V, Balaji S, Dustackeer VN, Narayanan PR. In silico analysis of mycobacteriophage Che12 genome: characterization of genes required to lysogenise Mycobacterium tuberculosis. Comput Biol Chem 2007; 31(2): 8291.

[12] Rybniker J, Kramme S, Small PL. Host range of 14 mycobacteriophages in Mycobacterium ulcerans and seven other mycobacteria including Mycobacterium tuberculosis--application for identification and susceptibility testing. J Med Microbiol 2006; 55(Pt 1): 37-42.

[13] Peng L, Chen BW, Luo YA, Wang GZ. Effect of mycobacteriophage to intracellular mycobacteria in vitro. Chin Med J (Engl) 2006; 119(8): 692-5.

[14] Sahu K, Gupta SK, Sau S, Ghosh TC. Comparative analysis of the base composition and codon usages in fourteen mycobacteriophage genomes. J Biomol Struct Dyn 2005; 23(1): 63-71.

[15] Ranjan A, Vidyarthi AS, Poddar R. Evaluation of codon bias perspectives in phage therapy of Mycobacterium tuberculosis by multivariate analysis. In Silico Biol 2007; 7(4-5): 423-31.

[16] Sharp PM, Li WH. The codon Adaptation Index--a measure of directional synonymous codon usage bias, and its potential applications. Nucleic Acids Res 1987; 15(3): 1281-95.

[17] Wright F. The 'effective number of codons' used in a gene. Gene 1990; 87(1): 23-9.

[18] Sharp PM, Cowe E. Synonymous codon usage in Saccharomyces cerevisiae. Yeast 1991; 7(7): 657-78.

[19] Greenacre MJ. Theory and applications of correspondence analysis. London: Academic Press 1984.

[20] Grantham R, Gautier C, Gouy M, Jacobzone M, Mercier R. Codon catalog usage is a genome strategy modulated for gene expressivity. Nucleic Acids Res 1981; 9(1): r43-74.

[21] Sharp PM, Rogers MS, McConnell DJ. Selection pressures on codon usage in the complete genome of bacteriophage T7. J Mol Evol 1984; 21(2): 150-60.

[22] Gouy M. Codon contexts in enterobacterial and coliphage genes. Mol Biol Evol 1987; 4(4): 426-44.

[23] Ikemura T. Transfer RNA in protein synthesis. Ann Arbor, Tokyo, London: CRC Press 1992.

[24] Zhou J, Liu WJ, Peng SW, Sun XY, Frazer I. Papillomavirus capsid protein expression level depends on the match between codon usage and tRNA availability. J Virol 1999; 73(6): 4972-82.

[25] Kanaya S, Yamada Y, Kinouchi M, Kudo Y, Ikemura T. Codon usage and tRNA genes in eukaryotes: correlation of codon usage diversity with translation efficiency and with CG-dinucleotide usage as assessed by multivariate analysis. J Mol Evol 2001; 53(45): $290-8$.

[26] Vasala. Characterization of Lactobacillus bacteriophage LL-H genes and proteins having biotechnological interest. Acta Universitatis Ouluensis A 315. Oulu: University of Oulu, 1998.

[27] Bailly-Bechet M, Vergassola M, Rocha E. Causes for the intriguing presence of tRNAs in phages. Genome Res 2007; 17(10): 1486-95.

[28] Brennan PJ. Structure, function, and biogenesis of the cell wall of Mycobacterium tuberculosis. Tuberculosis (Edinb) 2003; 83(1-3): $91-7$

[29] Minnikin DE. Lipids: complex lipids, their chemistry, biosynthesis and roles. In: The biology of mycobacteria, physiology, identification and classification. USA: Academic Press 1982; vol. 1: pp. 95-184.

[30] Summer EJ, Berry J, Tran TA, Niu L, Struck DK, Young R. $\mathrm{Rz} / \mathrm{Rz} 1$ lysis gene equivalents in phages of Gram-negative hosts. J Mol Biol 2007; 373(5): 1098-112. 
[31] Young R. Phage lysis. In phages: their role in bacterial pathogenesis and biotechnology. Washington, DC: American Society for Microbiology 2005; pp. 92-127.

[32] Arpigny JL, Jaeger KE. Bacterial lipolytic enzymes: classification and properties. Biochem J 1999; 343(Pt 1): 177-83.

[33] Gil F, Catalao MJ, Moniz-Pereira J, Leandro P, McNeil M, Pimentel M. The lytic cassette of mycobacteriophage Ms6 encodes an enzyme with lipolytic activity. Microbiology 2008; 154(Pt 5): 1364-71.

[34] Holmquist M. Alpha/Beta-hydrolase fold enzymes: structures, functions and mechanisms. Curr Protein Pept Sci 2000; 1(2): 20935 .

[35] Gupta R, Gupta N, Rathi P. Bacterial lipases: an overview of production, purification and biochemical properties. Appl Microbiol Biotechnol 2004; 64(6): 763-81.

(C) Hassan et al.; Licensee Bentham Open.

This is an open access article licensed under the terms of the Creative Commons Attribution Non-Commercial License (http: //creativecommons.org/licenses/by$\mathrm{nc} / 3.0 /$ ) which permits unrestricted, non-commercial use, distribution and reproduction in any medium, provided the work is properly cited. 